УДК 616.018

DOl: https://doi.org/10.17816/RCF194383-393

Обзорная статья

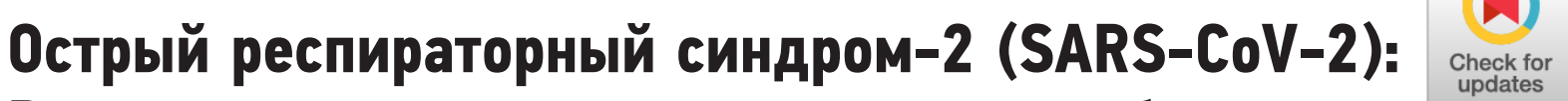 Раствор перекиси водорода и гидрокарбоната натрия как отхаркивающее средство для реканализации дыхательных путей и оксигенации крови при респираторной обструкции (обзор научной и патентной литературы)
}

\author{
А.Л. Ураков ${ }^{1}$, П.Д.Шабанов ${ }^{2,3}$ \\ ${ }^{1}$ Ижевская государственная медицинская академия, Ижевск, Россия; \\ 2 Институт экспериментальной медицины, Санкт-Петербург, Россия; \\ ${ }^{3}$ Военно-медицинская академия им. С.М. Кирова, Санкт-Петербург, Россия
}

Непосредственная причина смерти пациентов в финальной стадии новой коронавирусной инфекции — гипоксия, развивающаяся вследствие респираторной обструкции. В соответствии со стандартом лечения пациентов при наиболее тяжелой атипичной пневмонии при COVID-19 для сохранения их жизни применяется искусственная вентиляция легких и внелегочная оксигенация крови. Однако эти методы не устраняют обструкцию дыхательных путей, одной из причин которой является гиперсекреция слизи. В обзоре показано, что в России определен новый вектор поиска и разработки лекарственных средств для медикаментозного устранения гипоксии при респираторной обструкции. Показана высокая перспективность растворов перекиси водорода и гидрокарбоната натрия с оригинальными физикохимическими свойствами и локальными механизмами действия, обеспечивающими срочную реканализацию дыхательных путей и оксигенацию крови при респираторной обструкции, вызванной закупоркой дыхательных путей густой мокротой, слизью, гноем и кровью. Указываются отечественные изобретения, суть которых лежит в основе этого научного направления. Приводятся оригинальные рецептуры растворов перекиси водорода и гидрокарбоната натрия, а также новые технологии их локального применения, обеспечивающие срочное растворение слизи, мокроты, гноя и крови с одновременным немедленным выделением газа кислорода.

Ключевые слова: COVID-19; гипоксия; слизь; мокрота; гной; кислород; отхаркивающие средства.

\section{Как цитировать:}

Ураков А.Л., Шабанов П.Д. Острый респираторный синдром-2 (SARS-CoV-2): Раствор перекиси водорода и гидрокарбоната натрия как отхаркивающее средство для реканализации дыхательных путей и оксигенации крови при респираторной обструкции (обзор научной и патентной литературы) // Обзоры по клинической фармакологии и лекарственной терапии. 2021. Т. 19. № 4. C. 383-393. DOI: https://doi.org/10.17816/RCF194383-393 
Review

\title{
Acute respiratory syndrome-2 (SARS-CoV-2): A solution of hydrogen peroxide and sodium bicarbonate as an expectorant for recanalization of the respiratory tract and blood oxygenation in respiratory obstruction (review)
}

\author{
Aleksandr L. Urakov ${ }^{1}$, Petr D. Shabanov ${ }^{2,3}$ \\ ${ }^{1}$ Izhevsk State Medical Academy, Izhevsk, Russia; \\ ${ }^{2}$ Institute of Experimental Medicine, Saint Petersburg, Russia; \\ ${ }^{3}$ S.M. Kirov Military Medical Academy, Saint Petersburg, Russia
}

The immediate cause of death of patients in the final stage of a new coronavirus infection is hypoxia, which develops due to respiratory obstruction. In accordance with the standard of treatment of patients with the most severe atypical pneumonia with COVID-19, artificial lung ventilation and extrapulmonary blood oxygenation are used to preserve their lives. However, these methods do not eliminate airway obstruction, one of the causes of which is mucus hypersecretion. The review shows that a new vector for the search and development of medicines for the drug elimination of hypoxia in respiratory obstruction has been identified in Russia. The high prospects of solutions of hydrogen peroxide and sodium bicarbonate with original physicochemical properties and local mechanisms of action providing urgent recanalization of the respiratory tract and oxygenation of blood in respiratory obstruction caused by blockage of the respiratory tract with thick sputum, mucus, pus and blood are shown. Domestic inventions are indicated, the essence of which is the basis of this scientific direction. Original formulations of solutions of hydrogen peroxide and sodium bicarbonate are given, as well as new technologies for their local application, providing urgent dissolution of mucus, sputum, pus and blood with simultaneous immediate release of oxygen gas.

Keywords: COVID-19; hypoxia; mucus; sputum; pus; oxygen; expectorants.

To cite this article:

Urakov AL, Shabanov PD. Acute respiratory syndrome-2 (SARS-CoV-2): A solution of hydrogen peroxide and sodium bicarbonate as an expectorant for recanalization of the respiratory tract and blood oxygenation in respiratory obstruction (review). Reviews on Clinical Pharmacology and Drug Therapy. 2021;19(4):383-393. DOI: https://doi.org/10.17816/RCF194383-393 


\section{Гипоксия как следствие скопления слизи и гноя в просвете дыхательных путей, респираторной обструкции и как нерешенная проблема фармации и фармакологии}

Пандемия COVID-19 продолжает уносить жизни сотен тысяч пациентов на планете, несмотря на все успехи в фармации и фармакологии, а также несмотря на богатый арсенал лекарственных средств [1, 2]. Нет сомнений, что в финальной стадии болезни очень часто причина смерти пациентов связана с атипичной пневмонией, которая в заключительной стадии болезни осложняется острым респираторным синдромом-2 (SARS-CoV-2) и обструкцией дыхательных путей [3]. В свою очередь, обструкция дыхательных путей уменьшает внутрилегочную оксигенацию крови и вызывает гипоксию, которая постепенно прогрессирует и в тяжелой стадии может стать причиной смерти пациентов [4]. Поэтому именно газ кислород применяется в неотложных ситуациях для сохранения жизни пациентов при тяжелой гипоксии. При умеренной стадии гипоксии газ кислород вводят в систему дыхания в виде ингаляций с помощью искусственной вентиляции легких [5-8]. Затем, при тяжелой стадии атипичной пневмонии эффективность традиционной искусственной вентиляции легких снижается, поэтому приходится применять внелегочную оксигенацию крови [9]. Оба указанных способа оказания неотложной помощи достаточно эффективны, но эти способы применения газа кислорода не устраняют респираторную обструкцию, не восстанавливают воздушность легких и внутрилегочную оксигенацию крови. Иными словами, традиционная технология искусственной вентиляции легких и общепринятая технология внелегочной оксигенации крови имеют существенный недостаток - не устраняют причину гипоксии.

Показано, что при тяжелой стадии атипичной пневмонии, вызванной COVID-19, легкие теряют воздушность, в них развивается обструкция дыхательных путей, что визуализируется с помощью компьютерной томографии и широко используется для диагностики атипичной пневмонии [10-12]. Считается, что эти процессы формируются в основном при участии таких факторов, как воспаление и иммунитет [13]. Именно поэтому поиск эффективных лекарственных средств лечения тяжелой стадии COVID-19 продолжается в направлении воздействия на воспаление и иммунитет. В надежде найти эффективные лекарства для лечения COVID-19 исследователи всех стран тестируют противовирусные и иммуномодулирующие препараты [14, 15].

К сожалению, до сих пор не существует общепризнанных лекарственных препаратов для срочного устранения гипоксии при остром респираторном синдроме-2 (SARS-CoV-2). [16]. Показано, что вакцины не растворяют густую слизь, мокроту и гной, не увеличивают просвет дыхательных путей при обструктивном бронхите и обструкции дыхательных путей и не устраняют гипоксию $[17,18]$.
Именно поэтому вакцины не повышают воздушность легких и внутрилегочную оксигенацию крови, а также не применяются в лечении тяжелой атипичной пневмонии, вызванной COVID-19. Кроме этого, известные сыворотки, химиотерапевтические, противокашлевые, муколитические и отхаркивающие лекарственные средства также оказались мало эффективными при тяжелой стадии атипичной пневмонии [19].

В то же время антисептики и дезинфицирующие средства оказывают надежное противовирусное действие, но только при местном применении [14]. Поэтому антисептические и дезинфицирующие средства обоснованно применяют для профилактики пандемии COVID-19. Однако, несмотря на безусловную эффективность этих средств в профилактике COVID-19, их не используют для лечения этой новой коронавирусной инфекции и они не включены в стандарт лечения тяжелой атипичной пневмонии и респираторной обструкции.

Тем не менее в России при поиске и разработке средств для эффективной реканализации дыхательных путей и реоксигенации крови в условиях респираторной обструкции был сделан акцент на антисептик перекись водорода. Дело в том, что перекись водорода является не только антисептиком, но и средством, быстро растворяющим густые биологические массы, содержащие фермент каталазу, с одновременным выделением газа кислорода. Кроме этого, слизь, мокрота, гной и кровь могут скапливаться в просвете дыхательных путей изза затруднения их откашливания, что уменьшает воздушность легких и внутрилегочную оксигенацию крови. Следовательно, гиперсекреция слизи, скопление мокроты, гноя и крови может быть одной из причин развития гипоксии и смерти пациентов в финальной стадии тяжелой атипичной пневмонии при COVID-19. При этом известные отхаркивающие и муколитические лекарственные средства не устраняют респираторную обструкцию и не восстанавливают воздушность легких при атипичной пневмонии. Однако скопление слизи, мокроты и гноя в просвете дыхательных путей не привлекает внимание исследователей [20].

\section{Энтеральное и инъекционное введение растворов перекиси водорода оксигенирует кровь, обогащает организм кислородом и сохраняет жизнь в условиях гипоксии}

В условиях гипоксии традиционно используется газ кислород. Однако общепринятые технологии доставки кислорода к головному мозгу опираются не на лекарственные, а на механические способы. В настоящее время наиболее популярный способ оксигенации - это внелегочная оксигенация крови. Тем не менее лекарства тоже используются для лечения пациентов с гипоксией. В частности, способность защитить головной мозг от гипоксического повреждения при COVID-19 исследователи 
ищут при применении антигипоксантов, химиотерапевтических, антимикробных средств широкого спектра действия, противовоспалительных и иммуномодулирующих, антитромботических средств и витамина С [21]. Но несмотря на все усилия, задача срочной оксигенации головного мозга в условиях тяжелой гипоксии при COVID-19 не решена до сих пор.

Первые шаги по применению перекиси водорода для замены кислорода и продления жизни в условиях гипоксии были сделаны уже в начале XX в. Первые доказательства такой возможности были получены в России в опытах с аквариумными рыбками и донорской кровью. Результаты опытов показали, что рыбки и эритроциты легко усваивают кислород из перекиси водорода. Полученные результаты легли в основу двух изобретений: «Способ сохранения живой рыбы при транспортировке и хранении» (RU 2563151) и «Гипероксигенированное средство Е.М. Сойхер для насыщения венозной крови кислородом» (RU 2538662). Патенты на эти изобретения были получены в 2015 г.

Сущность первого изобретения заключается в том, что вместо кислорода в воду с плавающими рыбками периодически добавляют водный раствор перекиси водорода. Причем перекись водорода каждый раз добавляют не позднее, чем через 40 с после наступления чрезмерно повышенной двигательной активности рыбок. Было показано, что рыбки всасывают перекись водорода, которая затем распадается в крови на воду и газ кислород под действием фермента каталазы. И кислород легко усваивается организмом. Поэтому рыбки остаются живыми при полном отсутствии кислорода в воде, в которой они плавают, если в эту воду периодически вводить нужное количество перекиси водорода.

Сущность второго изобретения заключается в том, что в венозную кровь добавляют раствор 0,05-0,29\% перекиси водорода, который обеспечивает срочную инъекционную оксигенацию гемоглобина за счет выделения кислорода из перекиси водорода под действием фермента каталазы. В итоге темно-вишневый цвет венозной крови тут же изменяется на яркий красный цвет, характерный для артериальной крови.

Вслед за этим была показана возможность оксигенации крови при энтеральном и инъекционном введении раствора перекиси водорода. Эти данные легли в основу таких изобретений, как «Средство для повышения устойчивости к гипоксии» (RU 2604129) и «Лимфозаменитель для локального сохранения жизнеспособности органов и тканей при гипоксии и ишемии» (RU 2586292).

Первое средство представляет собой напиток, содержащий 0,3-0,5 \% перекиси водорода и газ кислород при избыточном давлении. Было показано, что энтеральное введение такого напитка повышает устойчивость организма к гипоксии за счет дополнительного вовлечения в газообмен слизистой оболочки желудка, что ведет к желудочной оксигенации крови.
Второе средство (лимфозаменитель) представляет собой раствор перекиси водорода и глюкозы, предназначенный для инъекций. Было показано, что инъекционное введение такого раствора в ткани продлевает их жизнеспособность при ишемии и гипоксии за счет оксигенации эритроцитов и/или клеток ткани. Оксигенация происходит за счет молекулярного кислорода, который появляется при расщеплении перекиси водорода под влиянием фермента каталазы. Кислород может связываться эритроцитами, либо использоваться иными клетками, включая клетки головного мозга, если инъекция осуществляется в головной мозг.

Затем была показана возможность оксигенации крови при введении раствора перекиси водорода в желудок (при энтеральном введении) [22]. Полученные результаты легли в основу двух изобретений: «Энергетический напиток» (RU 2639493) и «Средство для повышения физической выносливости» (RU 2634271). Патенты на эти изобретения были выданы в 2017 г. Изобретенные средства представляют собой пищевые напитки, которые содержат глюкозу, перекись водорода и газ кислород при избыточном давлении. При введении в желудок такие напитки оказываются в роли оживляющих средств за счет оксигенирующего действия перекиси водорода и газа кислорода одновременно. Дело в том, что перекись водорода и газ кислород всасываются в кровь, при этом перекись водорода расщепляется в крови под действием фермента каталазы на воду и газ кислород. Обогащение крови кислородом увеличивает оксигенацию крови.

Следовательно, энтеральное и/или инъекционное введение раствора перекиси водорода является дополнительным способом обогащения организма кислородом, так как перекись водорода легко всасывается в кровь, а кровь содержит фермент каталазу, которая тут же расщепляет перекись водорода на воду и газ кислород. Эритроциты усваивают кислород посредством оксигенации гемоглобина, что увеличивает оксигенацию крови, а это повышает устойчивость головного мозга к гипоксии. Все это позволяет рекомендовать инъекционное и энтеральное введение растворов перекиси водорода как перспективное направление для разработки медикаментозной внелегочной оксигенации крови в будущем.

\section{Теплый щелочной раствор перекиси водорода мгновенно превращает густой гной в пушистую кислородную пену}

Несмотря на то что патогенные микроорганизмы сохранили низкую устойчивость к местному денатурирующему действию антисептиков и дезинфицирующих средств, клиническая эффективность этих лекарственных средств при лечении гнойных болезней длительное время оставалась низкой [23-25]. Только в начале XX в. было установлено, что низкая эффективность антисептиков при лечении гнойных болезней объясняется отсутствием у известных лекарственных средств способности 
растворять густой гной. Оказалось, что при лечении гнойных заболеваний традиционно использовались препараты, оказывающие пагубное воздействие на все формы жизни, но не растворяющие гнойные массы [26].

Для выяснения возможности растворения гноя посредством известных антисептиков в 2005 г. были начаты исследования динамики вязкости густого гноя под влиянием местного действия различных лекарственных средств с учетом физико-химических факторов локального взаимодействия. Полученные результаты показали, что густой гной можно срочно растворять с помощью водных растворов антисептиков, обладающих такими физико-химическими свойствами, как щелочная, окислительная, осмотическая, температурная и газообразующая активность $[26,27]$. Эти данные легли в основу таких изобретений, как «Способ лечения эмпиемы плевры по Н.С. Стрелкову» (RU 2308894), «Способ маточного лаважа» (RU 2327471), «Средство для разжижения густого и липкого гноя» (RU 2360685) и «Гипергазированное и гиперосмотическое антисептическое средство» (RU 2331441).

В основе этих изобретений лежит локальная гипертермия и местное применение изотонических и/или гипертонических растворов $3 \%$ перекиси водорода с умеренной щелочной активностью, что обеспечивает присутствие 5-10 \% гидрокарбоната натрия. Дело в том, что введение такого раствора в кровь и/или гной тут же «запускает» бурную биохимическую реакцию расщепления перекиси водорода на воду и газ кислород. Этот процесс происходит под действием фермента каталазы, который всегда присутствует в крови и гное. При этом среда взаимодействия вспенивается и разрыхляется, превращаясь в эластичную пену белого цвета. Такой процесс образования пузырьков газа известен давно и именуется как «холодное кипение». Для усиления процесса холодного кипения было рекомендовано обогащать раствор перекиси водорода газом, вводя его под избыточным давлением, получая газированный раствор перекиси водорода аналогично газированным напиткам. С этой целью было предложено использовать углекислый газ, газ кислород и/или инертные газы. Было показано, что обогащенные газами щелочные растворы перекиси водорода обеспечивают более интенсивный процесс «холодного кипения», который очень эффективно растворяет и разрушает монолитную структуру густой гнойной массы.

Благодаря этим изобретениям была открыта новая подгруппа антисептических средств, названная «растворители гноя». Было показано, что «эталонный» растворитель густого гноя - это нагретый до температуры $37^{\circ} \mathrm{C}$ раствор $3 \%$ перекиси водорода, 10 \% натрия гидрокарбоната, содержащий газы (двуокись углерода, кислород или инертные газы типа гелия) под избыточным давлением 0,2-4 атм. При локальном взаимодействии такого раствора гной не просто растворяется, а почти мгновенно превращается в белую и очень мягкую кислородную пену белого цвета.
Вслед за этим были проведены исследования растворяющих возможностей щелочных растворов перекиси водорода при локальном взаимодействии с иными густыми биологическими тканями, содержащими фермент каталазу. В качестве таких тканей были использованы серные пробки, слезные камни, сгустки крови и зубной налет. Результаты исследований показали, что растворы перекиси водорода и гидрокарбоната натрия эффективно растворяют серные пробки, слезные камни и зубной налет при локальном взаимодействии. Полученные результаты легли в основу следующих изобретений: «Способ и средство для удаления серной пробки» (RU 2468776), «Многофункциональный раствор для эпибульбарных инстилляций» (RU 2452478) и «Средство для санации свищей при инфицированном панкреонекрозе» (RU 2455010). Было показано, что разработанные оригинальные растворы эффективно растворяют, разрушают и вспенивают серные пробки, густой гной и слезные камни. Растворение их достигается за счет щелочного омыления белково-липидных комплексов и внутритканевого процесса холодного кипения. При этом процесс холодного кипения происходит за счет выделения из перекиси водорода молекулярного газа кислорода под действием фермента каталазы, который всегда присутствует в гное, крови, слезных камнях и серных пробках.

В последующем аналогичные результаты были получены при локальном взаимодействии щелочных растворов перекиси водорода с зубным налетом. Полученные результаты легли в основу изобретения «Отбеливающий очиститель зубных протезов» (RU 2659952). Изобретенное средство - это водный раствор 2,0-10,0 \% натрия гидрокарбоната, $3 \pm 0,3 \%$ перекиси водорода и газа кислорода, который находится под избыточным давлением 0,2 атм. Используется этот раствор теплым $\left(37-42{ }^{\circ} \mathrm{C}\right)$. Было показано, что этот раствор очень быстро растворяет и вспенивает зубной налет, пятна крови, сгустки гноя, мокроту с прожилками крови за счет гипертермического размягчения, щелочного омыления, окислительного отбеливания, кавитационного разрыхления, флотации и суспензирования.

К этому следует добавить, что щелочные растворы перекиси водорода эффективно растворяют, обесцвечивают и превращают в белую пену сгустки крови. Это доказывают результаты серии опытов, полученные также в России. Полученные результаты лежат в основе следующих изобретений: «Способ экспресс-удаления пятен крови с одежды» (RU 2371532), «Отбеливатель кровоподтеков» (RU 2539380), «Отбеливающее средство» (RU 2589682), «Средство для внутрикожного отбеливания синяка» (RU 2573382), «Способ обесцвечивания кожи в области кровоподтека» (RU 2582215) и «Способ обесцвечивания кожи в области синяка» (RU 2586278). Все эти лекарственные средства представляют собой растворы, в которых основными ингредиентами являются 0,01-0,03 \% перекись водорода и 1,7 \% или 1,8 \% бикарбонат натрия. 
Их используют при температуре $37-42{ }^{\circ} \mathrm{C}$. Помимо этого, отбеливающая и пенообразующая активность щелочных растворов перекиси водорода при локальном взаимодействии с пятнами и следами крови лежит в основе таких изобретений, как «Способ экстренного отбеливания и удаления кровавой корочки с кожи на месте выдавленного угря» (RU 2631593), «Средство для прижизненного отбеливания кожи в области синяков под глазами» (RU 2639485), «Отбеливающий разрыхлитель высохшей крови для размачивания бинтов, прилипших к ране» (RU 2653465) и «Отбеливатель крови» (RU 2647371).

В качестве примера можно рассмотреть состав изобретенного отбеливателя крови. Это средство представляет собой насыщенный раствор гидрокарбоната натрия, в котором дополнительно содержится $3 \%$ перекиси водорода, используется теплым $\left(42{ }^{\circ} \mathrm{C}\right)$. Этот отбеливатель крови обеспечивает срочное обесцвечивание и удаление свежих и застарелых пятен крови с одежды, окровавленных бинтов, ватно-марлевых повязок, хирургических перчаток, медицинских инструментов, волос, кожи и слизистых оболочек в местах травм, ушибов, ссадин и хирургических разрезов. Следует подчеркнуть, что локальное взаимодействие проявляется не только обесцвечиванием и растворением пятен и сгустков крови, но и немедленным формированием из взаимодействующих сред эластичной кислородной пены белого цвета.

Помимо этого, указанные особенности местного действия щелочных растворов перекиси водорода на пятна и сгустки крови лежат в основе нескольких изобретений, обеспечивающих отличный эстетический результат в косметологии при гематомах. Речь идет о следующих изобретениях: «Способ отбеливания синяка под ногтем» (RU 2631592), «Способ лечения синего ногтя» (RU 2641386) и «Способ экстренного отбеливания кожной гематомы под глазом» (RU 2679334). Суть этих методов заключается в том, что раствор 3 \% перекиси водорода и $10 \%$ бикарбоната натрия при температуре $37-42{ }^{\circ} \mathrm{C}$ сначала вводят в полость гематомы в объеме, обеспечивающем обесцвечивание тканей, а затем этот раствор используют снаружи в качестве компресса.

Выявленная отбеливающая активность щелочных растворов перекиси водорода позволяет рекомендовать их как универсальные чистящие (отбеливающие) средства, для использования в дерматологии, косметологии и стоматологии. В частности, щелочной раствор перекиси водорода лежит в основе такого гигиенического средства, как «Способ применения раствора для удаления зубного налета с помощью ирригатора» (RU 2723138). Сущность этого изобретения сводится к удалению зубного налета с помощью ирригатора, в котором используется раствор 2,0-10,0 \% гидрокарбоната натрия и 2,7-3,3 \% перекиси водорода, который дополнительно содержит инертный газ аргон при избыточном давлении 3-4 атм. Причем указанный раствор хранится в герметично закрытом сосуде, а перед применением нагревается до температуры $43-65^{\circ} \mathrm{C}$.
Кроме этого, щелочные растворы перекиси водорода высоко эффективны и безопасны при мытье и чистке кухонной керамической, стеклянной посуды и других аналогичных изделий, которые могут быть запачканы следами и пятнами биологического происхождения, содержащими фермент каталазу [27]. В связи с этим щелочные растворы перекиси водорода получили еще одно название - «кислородообразующие чистящие средства».

Мы убеждены, что указанная область применения щелочного раствора перекиси водорода очень скоро будет расширена. Доказательством такой возможности являются результаты ее влияния на ороговевший слой эпидермиса на пятках ног людей. Оказалось, что щелочной раствор перекиси водорода эффективно растворяет эпидермис в области сухих мозолей. Полученные результаты легли в основу изобретения «Средство для пилинга при гиперкератозе стоп» (RU 2730451). Это средство представляет собой водный раствор, который содержит гидроксид калия, перекись водорода и газ кислород под избыточным давлением. Было показано, что применение этого средства нагретым до температуры $38-42{ }^{\circ} \mathrm{C}$ обеспечивает быстрое размягчение ороговевшего слоя эпидермиса в области сухих мозолей и подошвенной мозоли, размягчение избыточной некротической ноттевой пластины при грибковом поражении ногтей, восстановление эластичности, гладкости, цвета кожи, формы и цвета деформированной ногтевой пластины.

Таким образом, в России впервые в мире были разработаны лекарственные средства и медицинские технологии, обеспечивающие быстрое превращение в кислородную пену белого цвета таких биологических тканей, как густой гной, слезные камни, серные пробки, мокрота с прожилками крови, пятна и корочки крови, зубной налет и ороговевший слой эпидермиса. При локальном взаимодействии с биологическими тканями, содержащими фермент каталазу, такие щелочные растворы перекиси водорода вызывают биохимическое растворение вязких и плотных тканей за счет щелочного омыления их белково-липидных комплексов, физическое разрушение их монолитной структуры и гидродинамическое гейзер-подобное удаление наружу за счет внутритканевого холодного кипения, возникающего вследствие быстрого выделения пузырьков газа. Основными ингредиентами указанных средств служат вода, перекись водорода и гидрокарбонат натрия. Одна часть этих лекарственных средств получила название «растворители ноя», вторая часть - «отбеливатели кровоподтеков», третья — «кислородообразующие чистящие средства».

\section{Перекись водорода - легкодоступное безрецептурное антисептическое, отбеливающее, оксигенирующее, окисляющее и отхаркивающее средство}

Гиперсекреция слизи возможна при многих болезнях легких, не только при COVID-19 [28]. Кроме этого, нередко слизь может быть дополнена гноем и пятнами крови. 
Поэтому исследование новых возможностей срочной лекарственной замены слизи, мокроты и гноя внутри дыхательных путей на газ кислород с последующим увеличением внутрилегочной оксигенации крови остается очень актуальной задачей.

Показано, что традиционные муколитики, отхаркивающие лекарственные средства и общепринятые технологии их применения не обеспечивают срочную замену слизи на газ кислород внутри дыхательных путей [28-35]. В этих условиях единственным обоснованным направлением поиска и разработки новых лекарственных средств для реканализации дыхательных путей и внутрилегочной оксигенации крови при респираторной обструкции является модернизация изобретенных технологий локального применения щелочного раствора перекиси водорода. Дело в том, что щелочной раствор перекиси водорода при локальном взаимодействии с гноем, кровью либо со слизью и мокротой с прожилками крови (так же как с иными биологическими тканями, содержащими фермент каталазу) может быстро растворять и вспенивать их с образованием газа кислорода.

Перекись водорода относится к антисептикам, но в последние годы область ее применения была значительно расширена. Этому способствовало открытие у щелочного раствора перекиси водорода возможностей растворять ороговевший эпителий сухих мозолей, гной, зубной налет, слезные камни, серные пробки и сухие корочки крови. Нет противопоказаний к применению щелочных растворов перекиси водорода и в пульмонологии.

При этом установлено, что перекись водорода имеет следующие преимущества перед всеми иными известными лекарственными средствами.

1. Перекись водорода - это антисептик, который является известным противоинфекционным средством широкого спектра действия. Показано, что она оказывает противовирусное действие в полости рта и в системе дыхания, подавляя разные вирусы, включая коронавирусы [36]. Перекись водорода оказывает противобактериальное, противопротозойное и противогрибковое действие, что подтверждается высокой эффективностью ее применения в лечении гнойных ран на всех частях тела [37].

2. Перекись водорода - это одно из самых безопасных противоинфекционных безрецептурных лекарств, легко доступных населению [38-40].

3. Перекись водорода широко применяется в оториноларингологии и стоматологии как гигиеническое противовирусное средство [41, 42].

4. Перекись водорода растворяет и обесцвечивает густой гной, густую слизь, мокроту, зубной налет, сгустки, пятна и сухие корочки крови одновременно.

5. Перекись водорода быстро расщепляется под влиянием фермента каталазы на воду и газ кислород, «взрывает» гной и другие аналогичные биологические массы и превращает их в мягкую пену за счет бурного формирования множества пузырьков газа, вызывая процесс холодного кипения.

6. Перекись водорода в малых дозах оксигенирует эритроциты за счет оксигенации гемоглобина, а в больших дозах обесцвечивает гемоглобин и его цветные метаболиты, а также повышает локальную температуру за счет процессов окисления $[43,44]$.

Этим объясняется предложение щелочного раствора перекиси водорода для ингаляции при обструктивном бронхите с целью растворения слизи и гноя с одновременным увеличением внутрилегочной оксигенации крови. Суть этого изобретения защищена патентом на «Аэрозоль для ингаляций при обструктивном бронхите» (RU 2735502). Указанный аэрозоль обеспечивает размеры микрочастиц в диапазоне 0,5-2 мкм путем распыления жидкости или дисперсного распыления с помощью ультразвуковых, компрессионных и струйных ингаляторов и распылителей. Препарат содержит 1,2 \% бикарбоната натрия, 0,3-0,5 \% перекиси водорода и 0,5 \% гидрохлорида лидокаина при рН 8,5, осмотической активности 280-300 м0см/л воды и местной температуре 41-55 ${ }^{\circ} \mathrm{C}$. Показано, что аэрозоль через несколько секунд после начала вдоха почти полностью разрыхляет и растворяет густой гной и густую слизь в нижних бронхах, быстро способствует отхаркиванию мокроты и удушье исчезает на несколько часов [3].

Дополнительно к этому ингаляционное применение щелочного раствора перекиси водорода было предложено для лечения пациентов от COVID-19 на заключительном этапе. В 2021 г. в России был выдан патент на изобретение «Аэрозоль для инвазивной механической вентиляции легких при COVID-19» (RU № 2742505). Этот аэрозоль состоит из 2-10\% бикарбоната натрия, 0,3-0,5\% перекиси водорода, 0,5\% гидрохлорида лидокаина с рН 8,5, осмотической активностью 370-1990 м0см/л воды и местной температурой 37-55 C. Показано, что применение средства обеспечивает срочное устранение симптомов обструктивного бронхита, улучшение проходимости дыхательных путей для дыхательных газов, повышение эффективности оксигенации легочной крови при искусственной вентиляции легких и снижение степени гипоксии у пациентов интенсивной терапии. Первый реанимационный аэрозоль обеспечивает срочное растворение густого гноя и густой слизи в дыхательных путях, увеличивает содержание кислорода, устраняет отек слизистой оболочки, улучшает проходимость дыхательных путей для дыхательных газов при обструктивном бронхите и повышает оксигенацию крови во время искусственной вентиляции легких у реанимационных пациентов [45].

Кроме этого, в 2021 г. в России была разработана новая биологическая модель для скрининга лекарственных средств, способных в дыхательных путях срочно превращать слизь в кислородную пену. Для этой цели предлагается использовать изолированные 
легкие экспериментальных животных, а для имитации обструкции дыхательных путей была изобретена «Искусственная мокрота для имитации обструкции дыхательных путей при COVID-19» (RU 2748999). Рецептура искусственной мокроты включает 4,4-22,0 \% картофельного крахмала, 2,2-11,0 \% желатина, 0,9\% хлорида натрия и $5 \%$ крови сельскохозяйственного животного, разведенной дистиллированной водой в соотношении 1:1. Искусственная мокрота имеет $\mathrm{pH}$ 7,0-7,4, осмотическую активность 280-300 м0см/л воды и температуру $37{ }^{\circ} \mathrm{C}$. Такая искусственная мокрота обладает «необходимыми» физико-химическими и биохимическими свойствами. В частности, имеет «правильную» вязкость и содержит фермент каталазу, а также обеспечивает срочное субтотальное заполнение дыхательных путей изолированных легких экспериментальных животных. Поэтому такие легкие могут быть использованы в качестве биологической модели для скрининга новых лекарств. На сегодняшний день это единственная экспериментальная модель для поиска и разработки новых лекарственных средств, предназначенных для срочной реканализации дыхательных путей и внутрилегочной реоксигенации крови у пациентов с тяжелой неспецифической пневмонией, вызванной COVID-19.

\section{СПИСОК ЛИТЕРАТУРЫ}

1. Mahase E. COVID-19: reports from Israel suggest one dose of Pfizer vaccine could be less effective than expected // BMJ. 2021. Vol. 372. ID n217. DOl: 10.1136/bmj.n217

2. Harcourt J., Tamin A., Lu X., et al. Severe acute respiratory syndrome Coronavirus-2 from patient with coronavirus disease, United States // Emerg Infect Dis. 2020. Vol. 26. No. 6. P. 1266-1273. DOl: 10. 3201/eid2606.200516

3. Urakov A.L., Urakova N.A. COVID-19: Optimization of respiratory biomechanics by aerosol pus solvent // Russian Journal of Biomechanics. 2021. Vol. 25. No. 1. P. 86-90. DOI: 10.15593/RJBiomech/2021.1.07

4. Urakov A., Urakova N. Recent Insights into the Management of Inflammation in Asthma [letter] // J Inflamm Res. 2021. Vol. 14. P. 4603-4604. DOI: 10.2147/JIR.S337690

5. Oishee M.J., Ali T., Jahan N., et al. COVID-19 Pandemic: Review of Contemporary and Forthcoming Detection Tools // Infect Drug Resist. 2021. Vol. 14. P. 1049-1082. DOI: 10.2147/IDR.S289629

6. Urakov A.L., Urakova N.A. COVID-19: What drug can be used to treat a new coronavirus disease and why // J Bio Innov. 2020. Vol. 9. No. 3. P. 241-251. DOI: 10.46344/JBIN0.2020.v09i03.02

7. Urakov A., Urakova N. COVID-19. Cause of death and medication // IP Int J Comprehensive Adv Pharmacol. 2020. Vol. 5. No. 2. P. 45-48. DOI: 10.18231/j.ijcaap.2020.011

8. Fan E., Sorbo D.L., Goligher E.C., et al. An official American Thoracic / European Society of Intensive Care Medicine / Society of Critical Care Medicine clinical practice guideline: mechanical ventilation in adult patients with acute respiratory distress syndrome //

\section{ЗАКЛЮЧЕНИЕ}

Приведенный обзор изобретений, основанных на местном применении теплых щелочных растворов перекиси водорода, позволяет предположить, что сформированная в России стратегия поиска новых лекарственных средств для борьбы с респираторной обструкцией и гипоксией позволит в ближайшем будущем разработать новые препараты и методы их применения, которые могут стать достойной альтернативой экстракорпоральной мембранной оксигенации при тяжелой гипоксии и снизить смертность пациентов с COVID-19.

\section{ДОПОЛНИТЕЛЬНАЯ ИНФОРМАЦИЯ}

Вклад авторов. Все авторы подтверждают соответствие своего авторства международным критериям ICMJE (все авторы внесли существенный вклад в разработку концепции, проведение исследования и подготовку статьи, прочли и одобрили финальную версию перед публикацией).

Конфликт интересов. Авторы декларируют отсутствие явных и потенциальных конфликтов интересов, связанных с публикацией настоящей статьи.

Источник финансирования. Авторы заявляют об отсутствии внешнего финансирования при проведении исследования.

Am J Respir Crit Care Med. 2017. Vol. 195. No. 9. P. 1253-1263. DOI: 10.1164/rccm.201703-0548ST

9. Wunsch H. Mechanical ventilation in COVID-19: Interpreting the current epidemiology // Am J Respir Crit Care Med. 2020. Vol. 202. No. 1. P. 1-4. DOl: $10.1164 / \mathrm{rccm} .202004-1385 \mathrm{ED}$

10. Tay M.Z., Poh C.M., Rénia L., et al. The trinity of COVID-19: immunity, inflammation and intervention // Nat Rev Immunol. 2020. Vol. 20. No. 6. P. 363-374. D0I: 10.1038/s41577-020-0311-8

11. Yang W., Sirajuddin A., Zhang X., et al. The role of imaging in 2019 novel coronavirus pneumonia (COVID-19) // Eur Radiol. 2020. Vol. 30. No. 9. P. 4874-4882. D0I: 10.1007/s00330-020-06827-4

12. Li B., Li X., Wang Y., et al. Diagnostic value and key features of computed tomography in Coronavirus disease 2019 // Emerg Microbes Infect. 2020. Vol. 9. No. 1. P. 787-793. DOl: 10.1080/22221751.2020.1750307

13. Jacobi A., Chung M., Bernheim A., Eber C. Portable chest X-ray in coronavirus disease-19 (COVID-19): a pictorial review // Clin Imaging. 2020. Vol. 64. P. 35-42. DOl: 10.1016/j.clinimag.2020.04.001 14. Colson P., Rolain J.M., Lagier J.C., et al. Chloroquine and hydroxychloroquine as available weapons to fight COVID-19 // Int J Antimicrob Agents. 2020. Vol. 55. No. 4. ID105932. DOI: 10.1016/j.ijantimicag.2020.105932

15. Gautret P., Lagier J.C., Parola P., et al. Hydroxychloroquine and azithromycin as a treatment of COVID-19: results of an open-label non-randomized clinical trial // Int J Antimicrob Agents. 2020. Vol. 56. No. 1. ID105949. DOI: 10.1016/j.ijantimicag.2020.105949

16. Urakov A.L. COVID-19: Original simple and cheap extrapulmonary oxygenation as an alternative to ECMO // J Bio Innov. 2020. Vol. 9. No. 4. P. 648-654. DOI: 10.46344/JBIN0.2020.v09i04.27 
17. Nasir M., Zaman M.A., Majumder T.K., et al. Perception, Preventive Practice, and Attitude Towards Vaccine Against COVID-19 Among Health Care Professionals in Bangladesh // Infect Drug Resist. 2021. Vol. 14. P. 3531-3540. DOI: 10.2147/IDR.S326531

18. Mascellino M.T., Di Timoteo F., De Angelis M., Oliva A. Overview of the Main Anti-SARS-CoV-2 Vaccines: Mechanism of Action, Efficacy and Safety // Infect Drug Resist. 2021. Vol. 14. P. 3459-3476. DOI: 10.2147/IDR.S315727

19. Devaux C.A., Rolain J.M., Colson P., Raoult D. New insights on the antiviral effects of chloroquine against coronavirus: what to expect for COVID-19? // Int J Antimicrob Agents. 2020. Vol. 55. No. 5. ID105938. DOI: 10.1016/j.jjantimicag.2020.105938

20. Coronavirus and Pneumonia [internet]. [Дата обращения 02.11.2021]. Доступ по ссылке: https://www.webmd.com/lung/ covid-and-pneumonia\#1

21. Coronavirus Disease 2019 (COVID-19). Treatment Guidelines [internet]. Доступ по ссылке: https://www.covid19treatmentguidelines. nih.gov/

22. Urakov A.L. Hydrogen peroxide can replace gaseous oxygen to keep fish alive in hypoxia // Int Res J. 2017. No. 5. P. 106. DOI: 10.18454/IRJ.2227-6017

23. McDonnell G., Russell A.D. Antiseptics and Disinfectants: Activity, Action, and Resistance // Clin Microbiol Rev. 1999. Vol. 12. No. 1. P. 147-179. DOl: 10.1128/cmr.12.1.147

24. Sukovatykh B.S., Bezhin A.I., Pankrusheva T.A., et al. Treatment of purulent wounds immobilized antiseptics // Int J Pharm Sci Invent. 2016. Vol. 5. No. 7. P. 45-49. DOI: 10.18499/2070-478X-2017-10-4-269-276

25. Long R., Barrie J., Stewart K., Peloquin C.A. Treatment of a tuberculous empyema with simultaneous oral and intrapleural antituberculosis drugs // Can Respir J. 2008. Vol. 15. No. 5. P. 241-243. DOI: 10.1155/2008/747206

26. Ураков А.Л. Растворители гноя как новые лекарственные средства с уникальными физико-химическими свойствами // Обзоры по клинической фармакологии и лекарственной терапии. 2019. Т. 17, № 4. C. 89-95. DOI: 10.17816/RCF17489-95

27. Urakov A.L. Creation of "necessary" mixtures of baking soda, hydrogen peroxide and warm water as a strategy for modernization bleaching cleaners of ceramic // Epitőanyag - Journal of Silicate Based and Composite Materials. 2020. Vol. 72. No. 1. P. 30-35. DOI: 10.14382/epitoanyag-jsbcm.2020.6

28. Kurukulaaratchy R.J., Rupani H., Fong W.C.G., Kyyaly A. A Role for Mucolytics and Expectorants in Aiding Inhaled Therapies in Asthma? [response to letter] // J Inflamm Res. 2021. Vol. 14. P. 5183-5185. DOI: 10.2147/JIR.S341547

29. Bonser L.R., Erle D.J. Airway mucus and asthma: the role of MUC5AC and MUC5B // J Clin Med. 2017. Vol. 6. No. 12. P. 112. DOI: 10.3390/jcm6120112

30. Gibson P.G., Yang I.A., Upham J.W., et al. Effect of azithromycin on asthma exacerbations and quality of life in adults with persistent uncontrolled asthma (AMAZES): a randomised, double-blind, placebo-controlled trial // Lancet. 2017. Vol. 390. No. 10095. P. 659-668. DOI: 10.1016/S0140-6736(17)31281-3

31. Morinaga Y., Yanagihara K., Miyashita N., et al. Azithromycin, clarithromycin and telithromycin inhibit MUC5AC in- duction by Chlamydophila pneumoniae in airway epithelial cells // Pulm Pharmacol Ther. 2009. Vol. 22. No. 6. P. 580-586. DOI: 10.1016/j.pupt.2009.08.004

32. Poole P., Sathananthan K., Fortescue R. Mucolytic agents versus placebo for chronic bronchitis or chronic obstructive pulmonary disease // Cochrane Database Syst Rev. 2019. Vol. 2019. No. 5. ID CD001287. D0I: 10.1002/14651858.CD001287.pub6

33. Millman M., Millman F.M., Goldstein I.M., Mercandetti A.J. Use of acetylcysteine in bronchial asthma - another look // Ann Allergy. 1985. Vol. 54. No. 4. P. 294-296. PMID: 3985424.

34. Morgan L.E., Jaramillo A.M., Shenoy S.K., et al. Disulfide disruption reverses mucus dysfunction in allergic airway disease // Nat Commun. 2021. Vol. 12. No. 1. P. 249. DOl: 10.1038/s41467-020-20499-0

35. Juergens L.J., Worth H., Juergens U.R. New perspectives for mucolytic, anti-inflammatory and adjunctive therapy with 1,8-cineole in COPD and asthma: review on the new therapeutic approach // Adv Ther. 2020. Vol. 37. No. 5. P. 1737-1753. D0I: 10.1007/s12325-020-01279-0

36. Goyal S.M., Chander Y., Yezli S., Otter J.A. Evaluating the virucidal efficacy of hydrogen peroxide vapour // J Hosp Infect. 2014. Vol. 86. No. 4. P. 255-259. DOI: 10.1016/j.jhin.2014.02.003

37. Ionescu A.C., Brambilla E., Manzoli L., et al. Efficacy of personal protective equipment and $\mathrm{H}_{2} \mathrm{O}_{2}$-based spray against coronavirus in dental setting // Oral Dis. 2020. Online Version of Record before inclusion in an issue. DOI: 10.1111/odi.13736

38. Harris M.G., Gan C.M., Long D.A., Cushing L.A. The pH of over-the-counter hydrogen peroxide in soft lens disinfection systems // Optom Vis Sci. 1989. Vol. 66. No. 12. P. 839-842. DOI: 10.1097/00006324-198912000-00007

39. Safe and Effective Natural Remedy for the Flu: Over the Counter Hydrogen Peroxide [internet]. Доступ по ссылке: https://healthimpactnews.com/2018/safe-and-effective-natural-remedy-for-the-fluover-the-counter-hydrogen-peroxide/

40. Martin J.V., Sugawa C. Hydrogen peroxide ingestion with injury to upper gastrointestinal tract // World J Clin Cases. 2017. Vol. 5. No. 10. P. 378-381. D0I:10.12998/wjcc.v5.i10.378

41. Thejas S.R., Vinayak R., Sindu M. Hydrogen Peroxide as a Hemostatic Agent in Tonsillectomy: Is it Beneficial? // Saudi Journal of Otorhinolaryngology Head and Neck Surgery. 2021. Vol. 23. No. 1. P. 36-40. DOI:10.4103/sjoh.sjoh_40_20

42. Hydrogen Peroxide Uses for Skin, Mouth and Home [internet]. Доступ по ссылке: https://draxe.com/health/hydrogen-peroxide/

43. Urakov A.L., Stolyarenko A.P., Kopitov M.V., Bashirov I.I. Dynamics of the local temperature of blood, pus, mucus and catalase solution when they interact with a solution of hydrogen peroxide in vitro // Thermology International. 2021. Vol. 31. No. 3. P. 129-131.

44. Urakov A., Urakova N., Nikolenko V., et al. Current and emerging methods for treatment of hemoglobin related cutaneous discoloration: a literature review // Heliyon. 2021. Vol. 7. No. 1. ID e059542. DOI: 10.1016/j.heliyon.2021.e05954

45. Urakov A.L., Yagudin I.I., Suntsova D.0., et al. COVID-19: Thick Pus, Mucus and Sputum with Streaks of Blood as a Cause of Airway Obturation in SARS and Oxygen-Foaming Pus Solvent as a Medicine for their Recanalization // Acta Scientific Women's Health. 2021. Vol. 3. No. 5. P. 75-77. DOI: 10.31080/ASWH.2021.03.0221 


\section{REFERENCES}

1. Mahase E. COVID-19: reports from Israel suggest one dose of Pfizer vaccine could be less effective than expected. BMJ. 2021;372: ID n217. DOI: 10.1136/bmj.n217

2. Harcourt J, Tamin A, Lu X, et al. Severe acute respiratory syndrome Coronavirus 2 from patient with coronavirus disease, United States. Emerg Infect Dis. 2020;26(6):1266-1273. DOl: 10.3201/eid2606.200516

3. Urakov AL, Urakova NA. COVID-19: Optimization of respiratory biomechanics by aerosol pus solvent. Russian Journal of Biomechanics. 2021;25(1):86-90. DOI: 10.15593/RJBiomech/2021.1.07

4. Urakov A, Urakova N. Recent Insights into the Management of Inflammation in Asthma [letter]. J Inflamm Res. 2021;14:4603-4604. DOI: 10.2147/JIR.S337690

5. Oishee MJ, Ali T, Jahan N, et al. COVID-19 Pandemic: Review of Contemporary and Forthcoming Detection Tools. Infect Drug Resist. 2021;14:1049-1082. DOI: 10.2147/IDR.S289629

6. Urakov AL, Urakova NA. COVID-19: What drug can be used to treat a new coronavirus disease and why. J Bio Innov. 2020;9(3):241-251. DOI: 10.46344/JBIN0.2020.v09i03.02

7. Urakov A, Urakova N. COVID-19. Cause of death and medication. IP Int J Comprehensive Adv Pharmacol. 2020;5(2):45-48. DOI: 10.18231/j.ijcaap.2020.011

8. Fan E, Sorbo DL, Goligher EC, et al. An official American Thoracic / European Society of Intensive Care Medicine / Society of Critical Care Medicine clinical practice guideline: mechanical ventilation in adult patients with acute respiratory distress syndrome. Am J Respir Crit Care Med. 2017;195(9):1253-1263. DOI: 10.1164/rccm.201703-0548ST

9. Wunsch H. Mechanical ventilation in COVID-19: Interpreting the current epidemiology. Am J Respir Crit Care Med. 2020;202(1):1-4. DOI: 10.1164/rccm.202004-1385ED

10. Tay MZ, Poh CM, Rénia L, et al. The trinity of COVID-19: immunity, inflammation and intervention. Nat Rev Immunol. 2020;20(6):363-374. DOI: 10.1038/s41577-020-0311-8

11. Yang W, Sirajuddin A, Zhang $X$, et al. The role of imaging in 2019 novel coronavirus pneumonia (COVID-19). Eur Radiol. 2020;30(9):4874-4882. DOI: 10.1007/s00330-020-06827-4

12. Li B, Li X, Wang Y, et al. Diagnostic value and key features of computed tomography in Coronavirus disease 2019. Emerg Microbes Infect. 2020;9(1):787-793. DOI: 10.1080/22221751.2020.1750307

13. Jacobi A, Chung M, Bernheim A, Eber C. Portable chest X-ray in coronavirus disease-19 (COVID-19): a pictorial review. Clin Imaging. 2020;64:35-42. DOI: 10.1016/j.clinimag.2020.04.001

14. Colson P, Rolain JM, Lagier JC, et al. Chloroquine and hydroxychloroquine as available weapons to fight COVID-19. Int J Antimicrob Agents. 2020;55(4):105932. DOI: 10.1016/j.jjantimicag.2020.105932

15. Gautret $P$, Lagier JC, Parola $P$, et al. Hydroxychloroquine and azithromycin as a treatment of COVID-19: results of an openlabel non-randomized clinical trial. Int J Antimicrob Agents. 2020;56(1):105949. DOI: 10.1016/j.ijantimicag.2020.105949

16. Urakov AL. COVID-19: Original simple and cheap extrapulmonary oxygenation as an alternative to ECMO. J Bio Innov. 2020;9(4):648-654. DOI: 10.46344/JBIN0.2020.v09i04.27

17. Nasir M, Zaman MA, Majumder TK, et al. Perception, Preventive Practice, and Attitude Towards Vaccine Against COVID-19 Among Health Care Professionals in Bangladesh. Infect Drug Resist. 2021;14:3531-3540. DOI: 10.2147/IDR.S326531
18. Mascellino MT, Di Timoteo F, De Angelis M, Oliva A. Overview of the Main Anti-SARS-CoV-2 Vaccines: Mechanism of Action, Efficacy and Safety. Infect Drug Resist. 2021;14:3459-3476. D0I: 10.2147/IDR.S315727 19. Devaux CA, Rolain JM, Colson P, Raoult D. New insights on the antiviral effects of chloroquine against coronavirus: what to expect for COVID-19? Int J Antimicrob Agents. 2020;55(5):105938. DOI: 10.1016/j.ijantimicag.2020.105938

20. Coronavirus and Pneumonia [internet]. [Update 2021 November 2]. Available from: https://www.webmd.com/lung/covid-and-pneumonia\#1 21. Coronavirus Disease 2019 (COVID-19). Treatment Guidelines [internet]. Available from: https://www.covid19treatmentguidelines. nih.gov/

22. Urakov AL. Hydrogen peroxide can replace gaseous oxygen to keep fish alive in hypoxia. Int Res J. 2017;(5):106. DOI: 10.18454/IRJ.2227-6017

23. McDonnell G, Russell AD. Antiseptics and Disinfectants: Activity, Action, and Resistance. Clin Microbiol Rev. 1999;12(1):147-179. DOI: 10.1128/cmr.12.1.147

24. Sukovatykh BS, Bezhin Al, Pankrusheva TA, et al. Treatment of purulent wounds immobilized antiseptics. Int J Pharm Sci Invent. 2016;5(7):45-49. DOI: 10.18499/2070-478X-2017-10-4-269-276

25. Long R, Barrie J, Stewart K, Peloquin CA. Treatment of a tuberculous empyema with simultaneous oral and intrapleural antituberculosis drugs. Can Respir J. 2008;15(5):241-243. DOI: 10.1155/2008/747206.

26. Urakov AL. Pus solvents as new drugs with unique physical and chemical property. Reviews on Clinical Pharmacology and Drug Therapy. 2019;17(4):89-95. (In Russ.) DOI: 10.17816/RCF17489-95

27. Urakov AL. Creation of "necessary" mixtures of baking soda, hydrogen peroxide and warm water as a strategy for modernization bleaching cleaners of ceramic. Epitőanyag - Journal of Silicate Based and Composite Materials. 2020;72(1):30-35. DOI: 10.14382/epitoanyag-jsbcm.2020.6

28. Kurukulaaratchy RJ, Rupani H, Fong WCG, Kyyaly A. A Role for Mucolytics and Expectorants in Aiding Inhaled Therapies in Asthma? [Response to letter]. J Inflamm Res. 2021;14:5183-5185. DOI: $10.2147 / J I R . S 341547$

29. Bonser LR, Erle DJ. Airway mucus and asthma: the role of MUC5AC and MUC5B. J Clin Med. 2017;6(12):112. DOI: 10.3390/jcm6120112

30. Gibson PG, Yang IA, Upham JW, et al. Effect of azithromycin on asthma exacerbations and quality of life in adults with persistent uncontrolled asthma (AMAZES): a randomised, doubleblind, placebo-controlled trial. Lancet. 2017;390(10095):659-668. DOl: 10.1016/S0140-6736(17)31281-3

31. Morinaga Y, Yanagihara K, Miyashita N, et al. Azithromycin, clarithromycin and telithromycin inhibit MUC5AC induction by Chlamydophila pneumoniae in airway epithelial cells. Pulm Pharmacol Ther. 2009;22(6):580-586. DOI: 10.1016/j.pupt.2009.08.004

32. Poole P, Sathananthan K, Fortescue R. Mucolytic agents versus placebo for chronic bronchitis or chronic obstructive pulmonary disease. Cochrane Database Syst Rev. 2019;2019(5): CD001287. DOI: 10.1002/14651858.CD001287.pub6

33. Millman M, Millman FM, Goldstein IM, Mercandetti AJ. Use of acetylcysteine in bronchial asthma - another look. Ann Allergy. 1985;54(4):294-296. PMID: 3985424. 
34. Morgan LE, Jaramillo AM, Shenoy SK, et al. Disulfide disruption reverses mucus dysfunction in allergic airway disease. Nat Commun. 2021;12(1):249. DOI: 10.1038/s41467-020-20499-0

35. Juergens LJ, Worth $H$, Juergens UR. New perspectives for mucolytic, anti-inflammatory and adjunctive therapy with 1,8-cineole in COPD and asthma: review on the new therapeutic approach. $A d v$ Ther. 2020;37(5):1737-1753. DOI: 10.1007/s12325-020-01279-0

36. Goyal SM, Chander Y, Yezli S, Otter JA. Evaluating the virucidal efficacy of hydrogen peroxide vapour. J Hosp Infect. 2014;86(4):255-259. DOl: 10.1016/j.jhin.2014.02.003

37. Ionescu AC, Brambilla E, Manzoli L, et al. Efficacy of personal protective equipment and $\mathrm{H}_{2} \mathrm{O}_{2}$-based spray against coronavirus in dental setting. Oral Dis. 2020. Online Version of Record before inclusion in an issue. DOl: 10.1111/odi.13736

38. Harris MG, Gan CM, Long DA, Cushing LA. The pH of over-thecounter hydrogen peroxide in soft lens disinfection systems. Optom Vis Sci. 1989;66(12):839-842. D0l: 10.1097/00006324-198912000-00007 39. Safe and Effective Natural Remedy for the Flu: Over the Counter Hydrogen Peroxide [internet]. Available from: https://healthimpactnews.com/2018/safe-and-effective-natural-remedy-for-the-fluover-the-counter-hydrogen-peroxide/

\section{ОБ АВТОРАХ}

*Александр Ливиевич Ураков, д-р мед. наук, профессор; адрес: Россия, 426034, Ижевск, ул. Коммунаров, д. 281; ORCID: https://orcid.org/0000-0002-9829-9463;

eLibrary SPIN: 1613-9660; e-mail: urakoval@live.ru.

Петр Дмитриевич Шабанов, д-р мед. наук, профессор; ORCID: https://orcid.org/0000-0003-1464-1127; eLibrary SPIN: 8974-7477; e-mail: pdshabanov@mail.ru.
40. Martin JV, Sugawa C. Hydrogen peroxide ingestion with injury to upper gastrointestinal tract. World J Clin Cases. 2017;5(10):378-381. DOI: 10.12998/wjcc.v5.i10.378

41. Thejas SR, Vinayak R, Sindu M. Hydrogen Peroxide as a Hemostatic Agent in Tonsillectomy: Is it Beneficial? Saudi Journal of Otorhinolaryngology Head and Neck Surgery. 2021;23(1):36-40. DOI: 10.4103/sjoh.sjoh_40_20.

42. Hydrogen Peroxide Uses for Skin, Mouth and Home [internet]. Available from: https://draxe.com/health/hydrogen-peroxide/

43. Urakov AL, Stolyarenko AP, Kopitov MV, Bashirov II. Dynamics of the local temperature of blood, pus, mucus and catalase solution when they interact with a solution of hydrogen peroxide in vitro. Thermology International. 2021;31(3):129-131.

44. Urakov A, Urakova N, Nikolenko V, et al. Current and emerging methods for treatment of hemoglobin related cutaneous discoloration: a literature review. Heliyon. 2021;7(1):e059542. DOI: 10.1016/j.heliyon.2021.e05954

45. Urakov AL, Yagudin II, Suntsova DO, et al. COVID-19: Thick Pus, Mucus and Sputum with Streaks of Blood as a Cause of Airway Obturation in SARS and Oxygen-Foaming Pus Solvent as a Medicine for their Recanalization. Acta Scientific Women's Health. 2021;3(5): 75-77. DOI: 10.31080/ASWH.2021.03.0221 